\title{
Editorial
}

Most of those people who attended the XXI World's Poultry Congress in Montréal left with a sense of accomplishment and fulfilment. Those of us who had spent eight years organising the Congress left with a sense of relief, secure in the belief that our efforts had been fruitful. One of the innovations at the XXI Congress was the presentation of the Proceedings only in the CD-ROM format. While this is not new technology, its use as the exclusive medium for Congress Proceedings is relatively untried. Although we attempted to provide as flexible a system as possible, we have been made aware that some people have not been successful in retrieving the information from the CDROM. The search engine incorporated into the CD-ROM requires Windows 98 and Microsoft Word. We have found that, with this software, it works very well. For earlier and later versions of Windows, software can be downloaded from the Internet (see below). Other critics have asked how university and institutional libraries can access the Proceedings. We will provide CD-ROMs free of charge to them, on request. To make access to the Proceedings of the XXI Congress more widespread, the entire document has been posted to the web sites of both the Congress [www.wpc2000.org] and the WPSA [www.wpsa.com]. A search engine similar to the one used on the CDROM is incorporated so that visitors to the sites can search the index and download the required papers. We feel that this now provides equivalent or better access, at a much lower cost to the Congress, than could have been achieved using printed Proceedings. The world is moving rapidly towards electronic communications in many spheres. It is likely that, within a year, this journal will be available on the worldwide web. We are currently investigating the virtue of placing the WPSJ in Science Direct, a database with over 1500 scientific journals maintained by Elsevier. This would not only improve access by scientists around the world; it ultimately saves the Association money when members or subscribers choose to use only the electronic form. So, along with the poultry industry, our Association is quickly adapting to the electronic communications revolution. While some may regret the demise of the paper medium, it is clear that, for reasons of volume alone, modern information storage and transmission demands use of the latest technology.

As your incoming President, you are entitled to know of my plans for the Association and the journal for the next four years. I hope to build on the work of our past two Presidents, Mrs Desai and Dr Simons, in the initiation of new branches. The WPSA now has more branches than ever before, but we are still poorly represented in South and Central America. We are working hard to correct this and hope to welcome some new branches in the next year. The WPSA has long been an advocate of genetic conservation. This was highlighted in Don Shaver's address at the Opening Ceremony in Montréal. There are two aspects to this issue. One is the diminishing number of native and pure breeds around the world. The FAO is endeavouring to catalogue these and the WPSA will assist in this effort where possible. Secondly, with the number of commercial breeders continuing to decline through mergers and amalgamations, the genetic base for the world's commercial poultry continues to narrow. We hope to address both aspects in our continuing work with the FAO and others. I hope that our journal will continue to publish the high quality and variety of articles which have helped to establish its reputation in the scientific community.

\section{Peter Hunton}

II am delighted that the new President of the Association has quickly decided to follow the lead pioneered by Dietmar Flock and taken the opportunity to share his aspirations for the WPSA with you. Ed.] 